\title{
Electromagnetic fields stress living cells
}

\author{
Martin Blank $^{\mathrm{a}, *}$, Reba Goodman ${ }^{\mathrm{b}}$ \\ a Department of Physiology, Columbia University, New York, NY, USA \\ b Department of Pathology, Columbia University, New York, NY, USA
}

Received 30 January 2009; accepted 30 January 2009

\begin{abstract}
Electromagnetic fields (EMF), in both ELF (extremely low frequency) and radio frequency (RF) ranges, activate the cellular stress response, a protective mechanism that induces the expression of stress response genes, e.g., HSP70, and increased levels of stress proteins, e.g., hsp70. The 20 different stress protein families are evolutionarily conserved and act as 'chaperones' in the cell when they 'help' repair and refold damaged proteins and transport them across cell membranes. Induction of the stress response involves activation of DNA, and despite the large difference in energy between ELF and RF, the same cellular pathways respond in both frequency ranges. Specific DNA sequences on the promoter of the HSP70 stress gene are responsive to EMF, and studies with model biochemical systems suggest that EMF could interact directly with electrons in DNA. While low energy EMF interacts with DNA to induce the stress response, increasing EMF energy in the RF range can lead to breaks in DNA strands. It is clear that in order to protect living cells, EMF safety limits must be changed from the current thermal standard, based on energy, to one based on biological responses that occur long before the threshold for thermal changes.
\end{abstract}

(C) 2009 Elsevier Ireland Ltd. All rights reserved.

Keywords: DNA; Biosynthesis; Electromagnetic fields; ELF; RF

\section{Electromagnetic fields (EMF) alter protein synthesis}

Until recently, genetic information stored in DNA was considered essentially invulnerable to change as it was passed on from parent to progeny. Mutations, such as those caused by cosmic radiation at the most energetic end of the EM spectrum, were thought to be relatively infrequent. The model of gene regulation was believed to be that the negatively charged DNA was tightly wrapped up in the nucleus with positively charged histones, and that most genes were 'turned off' most of the time. Of course, different regions of the DNA code are being read more or less all the time to replenish essential

Abbreviations: EMF, electromagnetic fields; Hz, hertz; ELF, extremely low frequency; RF, radio frequency; MAPK, mitogen activated protein kinase; ERK1 $\backslash 2$, extracellular signal regulated kinase; JNK, c-Jun-terminal kinase p38MAPK; SAPK, stress activated protein kinase; NADH, nicotinamide adenine dinucleotide dehydrogenase; ROS, reactive oxygen species.

* Corresponding author at: Department of Physiology, Columbia University, 630 West 168 Street, New York, NY 10032,

USA. Tel.: +1 212305 3644; fax: +1 2123055775 .

E-mail address: mb32@ columbia.edu (M. Blank). proteins that have broken down and those needed during cell division.

New insights into the structure and function of DNA have resulted from numerous, well-done laboratory studies. The demonstration that EMF induces gene expression and the synthesis of specific proteins [1,2] generated considerable controversy from power companies, government agencies, physicists, and most recently, cell phone companies. Physicists have insisted that the reported results were not possible because there was not enough energy in the power frequency range (ELF) to activate DNA. They were thinking solely of mechanical interaction with a large molecule and not of the large hydration energy tied up in protein and DNA structures that could be released by small changes in charge [3]. Of the biologists who accepted such results [4], most thought that the EMF interaction originated at, and was amplified by, the cell membrane and not with DNA.

It is now generally accepted that weak EMF in the power frequency range can activate DNA to synthesize proteins. An EMF reactive sequence in the DNA has been identified [5] and shown to be transferable to other gene promoters [6]. This DNA sequence acts as an EMF sensitive antenna 


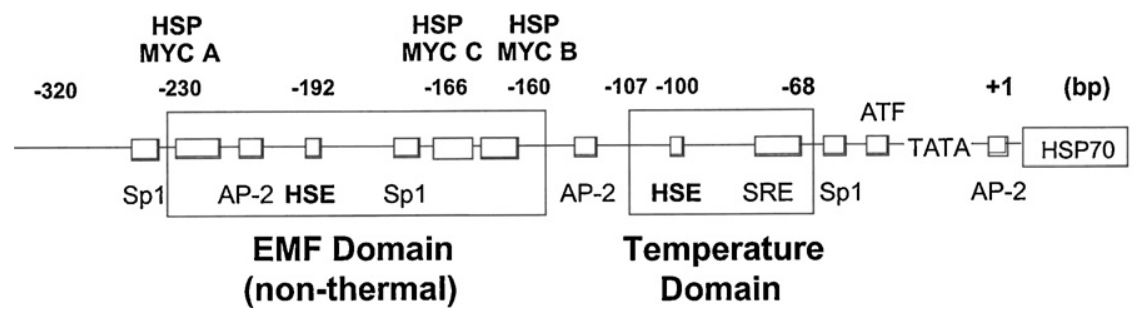

Fig. 1. Diagram of the HSP70 promoter showing the two different DNA sequences that have been identified as activated by EMF (non-thermal) and by thermal stimuli, respectively. The EMF domain contains three nCTCTn consensus sequences (electromagnetic response elements; EMRE), and differs from the consensus sequence (nGAAn) in the temperature or thermal domain.

that responds to EMF when transfected into reporter genes. Research at the more energetic levels of power frequency [7] and in the RF [8] ranges has shown that exposure to EMF can lead to breaks in the DNA strands. Therefore, DNA can no longer be considered unaffected by environmental EMF levels. It can be activated and damaged by EMF at levels that are considered safe [9]. The vulnerability of DNA to environmental influences and the possible dangers associated with EMF, had been underscored by discovery of EMF activation of the cellular stress response in the ELF range $[10,11]$. The cellular stress response is an unambiguous signal by the cell that EMF is potentially harmful.

\section{Physiological stress and cellular stress}

Discussions of physiological stress mechanisms usually describe responses of the body to pain, fear, 'oxygen debt' from muscle overexertion. These responses are mediated by organ systems. For example, the nervous system transmits action potentials along a network of nerves to cells, such as adrenal glands, that release rapidly acting agents such as epinephrine and norepinephrine and slower acting mineralocorticoids. These hormones are transported throughout the body by the circulatory system. They mobilize the defenses to cope with the adverse conditions and enable the body to 'fight or flee' from the noxious stimuli. The defensive actions include changes in heart rate, breathing rate, muscle activity, etc.

In addition to the responses of organ systems, there are protective mechanisms at the cellular level known as the cellular stress response. These mechanisms are activated by damage to cellular components such as DNA and protein [12], and the responses are characterized by increased levels of stress proteins [13] indicating that stress response genes have been upregulated in response to the stress.

The first stress response mechanism identified was the cellular reaction to sharp increases in temperature [14] and was referred to as 'heat shock', a term that is still retained in the nomenclature of the protective proteins, the hsps, heat shock proteins. Stress proteins are designated by the prefix 'hsp' followed by a number that gives the molecular weight in kilodaltons. There are about 20 different protein families ranging in molecular weight from a few kilodaltons to over
$100 \mathrm{kD}$, with major groups of proteins around $30 \mathrm{kD}, 70 \mathrm{kD}$ and $90 \mathrm{kD}$.

Research on the 'heat shock' response has shown that hsp synthesis is activated by a variety of stresses that are potentially harmful to cells, including physical stimuli like $\mathrm{pH}$ and osmotic pressure changes, as well as chemicals such as alcohol and toxic metal ions like $\mathrm{Cd}^{2+}$. EMF is a recent addition to the list of physical stimuli. It was initially shown in the power frequency (extremely low frequency, ELF) range [13], but shortly afterwards, radio frequency (RF) fields [15] and amplitude modulated RF fields [16] were shown to activate the same stress response.

Studies of stress protein stimulation by low frequency EMF have focused on a specific DNA sequence in the gene promoter that codes for hsp70, a major stress protein. Synthesis of this stress protein is initiated in a region of the promoter (see Fig. 1) where a transcription factor known as heat shock factor 1 (HSF-1) binds to a heat shock element (HSE). This EMF sensitive region on the HSP70 promoter is upstream from the thermal domain of the promoter and is not sensitive to increased temperature. The binding of HSF-1 to HSE occurs at -192 in the HSP70 promoter relative to the transcription initiation site. The EMF domain contains three nCTCTn myc-binding sites -230 , -166 and -160 relative to the transcription initiation site and upstream of the binding sites for the heat shock (nGAAn) and serum responsive elements $[5,6,17,18]$. The electromagnetic response elements (EMREs) have also been identified on the c-myc promoter and are also responsive to EMF. The sensitivity of the DNA sequences, nCTCTn, to EMF exposures has been demonstrated by transfecting these sequences into CAT and Luciferase reporter genes [6]. Thus, the HSP70 promoter contains different DNA regions that are specifically sensitive to different stressors, thermal and non-thermal.

Induction of increased levels of the major stress protein, hsp70, by EMF is rapid, within $5 \mathrm{~min}$. Also it occurs at extremely low levels of energy input, 14 orders of magnitude lower than with a thermal stimulus [10]. The far greater sensitivity to EMF than to temperature change in elevating the protective protein, hsp70, has been demonstrated to have potential clinical application, preventing injury from ischemia reperfusion [19-21]. George et al. [22] have shown the non-invasive use of EMF-induced stress proteins improved hemodynamic parameters during reperfusion 

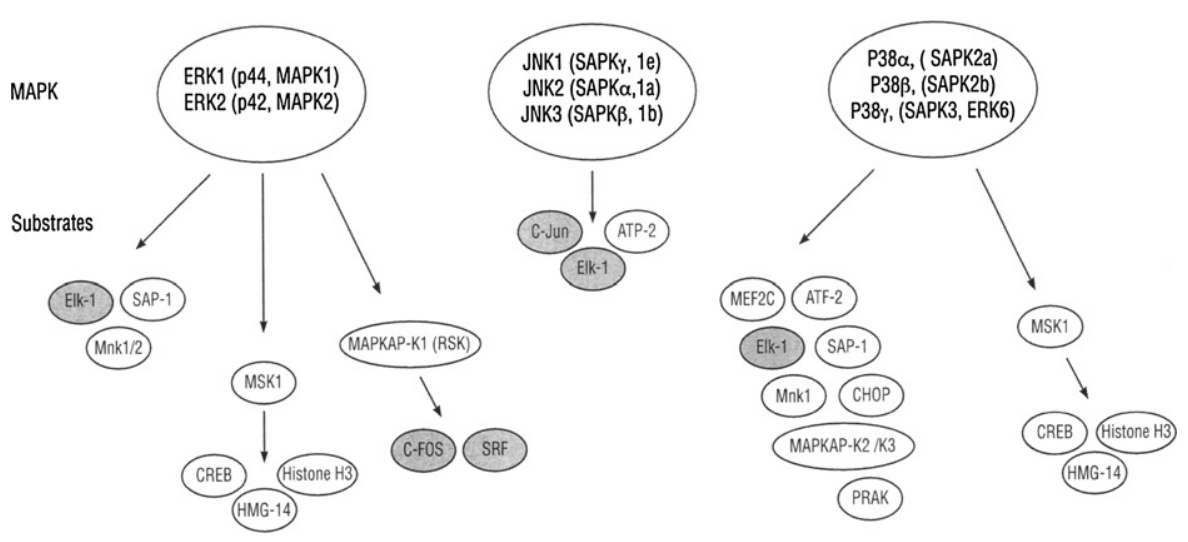

Fig. 2. The four mitogen activated protein kinase (MAPK) signaling cascades identified to date are: extracellular signal regulated kinase 1/2 (ERK), c-Junterminal kinase (JNK), p38MAPK and stress activated protein kinase (SAPK). Elements of the three MAPkinase pathways that have been identified as activated by EMF are shown as the shaded circles.

following ischemia. This effect occurred in the absence of measurable increased temperature.

\section{EMF interaction with signaling pathways}

EMF penetrate cells unattenuated and so can interact directly with the DNA in the cell nucleus, as well as other cell constituents. However, biological agents are impeded by membranes and require special mechanisms to gain access to the cell interior. Friedman et al. [23] have demonstrated that the initial step in transmitting extracellular information from the plasma membrane to the nucleus of the cell occurs when NADH oxidase rapidly generates reactive oxygen species (ROS). These ROS stimulate matrix metalloproteinases that allow them to cleave and release heparin binding epidermal growth factor. This secreted factor activates the epidermal growth receptor, which in turn activates the extracellular signal regulated kinase $1 \backslash 2$ (ERK) cascade. The ERK cascade is one of the four mitogen-activated protein kinase (MAPK) signaling cascades that regulate transcriptional activity in response to extracellular stimuli. The elements of the three

\section{EMF $\leftarrow$ DNA $\rightarrow$ hsp70 $\measuredangle$ Ros $\quad \downarrow \uparrow ? \quad \downarrow \uparrow ?$ Signaling Pathways}

Fig. 3. The signaling pathways and the stress response are activated by EMF. The activation mechanisms discussed in the text are indicated by arrows. In the stress response, DNA activation leads to hsp synthesis and may be due to direct EMF interaction with DNA. The signaling pathways are activated by reactive oxygen species (ROS) that are probably generated by EMF. Possible interactions between the pathways, DNA and hsp are indicated with question marks. In any case, EMF leads to activation of all the processes shown.
MAPK signaling cascades implicated in exposures to ELF and RF are highlighted in Fig. 2.

The four MAPK cascades are: (1) ERK, (2) c-Jun-terminal kinase (JNK), (3) stress activated protein kinase (SAPK) and (4) p38SAPK. Each of the cascades is composed of three to six tiers of protein kinases, and their signals are transmitted by sequential phosphorylation and activation of the protein kinases in each of the tiers. The result is activation of a large number of regulatory proteins, which include a set of transcription factors, e.g., c-Jun, c-Fos, hsp27 and hsp70. Activation of the stress response is accompanied by activation of specific signal transduction cascades involved in regulating cell proliferation, differentiation and metabolism [24-26]. The MAPK pathways have been characterized in several cell types [24,27-30]. Exposure to non-thermal ELF as well as thermal RF affects the expression of many cellular proteins [23-25] (Fig. 3).

The elevated expression of these protein transcription factors participate in the induction of various cellular processes, including several that are affected by cell phones, e.g., replication and cell-cycle progression [25,31] and apoptosis [32]. RF fields have been shown to activate specific transcription factor binding that stimulate cell proliferation and induce stress proteins [25,33]. It has been reported [31] that within 10 min of cell phone exposures, two MAPKinase cascades, p38 and ERK $1 \backslash 2$, are activated. Both ELF and RF activate the upregulation of the HSP70 gene and induction of elevated levels of the hsp70 protein. This effect on RNA transcription and protein stability is controlled by specific protein transcription factors that are elements of the mitogen MAPK cascade.

EMF also stimulate serum response factor which binds to the serum response element (SRE) through ERK MAPK activation and is associated with injury and repair in vivo and in vitro. The SRE site is on the promoter of an early response gene, c-fos, which under specific cellular circumstances has oncogenic properties. The c-fos promoter is EMF-sensitive; a $20 \mathrm{~min}$ exposure to $60 \mathrm{~Hz} 80 \mathrm{mG}$ fields significantly increases c-fos gene expression [34]. The SRE accessory protein, 
Elk-1, contains a growth-regulated transcriptional activation domain. ERK phosphorylation potentiates Elk-1 and transactivation at the c-fos SRE [29].

During the past twenty years, the growing use of cellular phones has aroused great concern regarding the health effects of exposure of the brain to $900 \mathrm{MHz}$ RF waves. Despite claims that the energy level is too low to induce changes in DNA and that the devices are safe, the non-thermal effects that have been demonstrated at both ELF and RF exposure levels can cause physiological changes in cells and tissues even at the level of DNA. Finally, it should be mentioned that some of the pathways described in this section also have roles in protein synthesis via RNA polymerase III, an enzyme in oncogenic pathways [35] and could, therefore, provide a mechanistic link between cancer and EMF exposure.

\section{Cells affected by the stress response}

Reviews on EMF and the stress response have appeared for the ELF range [13] and for the RF range [36]. The most recent review was published online in section 7 of the Bioinitiative Report [9], and it summarized both ELF and RF studies, mainly at frequencies $50 \mathrm{~Hz}, 60 \mathrm{~Hz}, 900 \mathrm{MHz}$ and $1.8 \mathrm{GHz}$. The citations in that review were not exhaustive, but the different frequencies and biological systems represent the diversity of results on stimulation of DNA and stress protein synthesis in many different cells. It is clear that the stress response does not occur in reaction to EMF in all types of cells, and sometimes because of the use of tissue cultured cell lines, even the same cell line can give opposite results in the same laboratory [37].

Many different types of cells have been shown to respond to EMF, both in vivo and in vitro, including epithelial, endothelial and epidermal cells, cardiac muscle cells, fibroblasts, yeast, E. coli, developing chick eggs, and dipteran cells (see Bioinitiative Report [9], section 7). Tissue cultured cells are less likely to show an effect of EMF, probably because immortalized cells have been changed significantly to enable them to live indefinitely in unnatural laboratory conditions. This may also be true of cancer cells, although some (e.g., MCF7 breast cancer cells) have responded to $\operatorname{EMF}[38,39]$, and in HL60 cells, one cell line responds to EMF while another does not [24]. Czyz et al. [16] found that p53-deficient embryonic stem cells showed an increased EMF response, but the wild type did not.

A broad study of genotoxic effects (i.e., DNA damage) in different kinds of cells [40] found no effects with lymphocytes, monocytes and skeletal muscle cells, but did find effects with fibroblasts, melanocytes and rat granulosa cells. Other studies [41,42] have also found that the blood elements, such as lymphocytes and monocytes are natural cells that have not responded. Since mobile cells can easily move away from a stress, there would be little selective advantage and evolutionary pressure for developing the stress response. The lack of response by skeletal muscle cells is related to the need
Table 1

Biological thresholds in the ELF range.

\begin{tabular}{|c|c|c|}
\hline Biological system & $\begin{array}{l}\text { Threshold } \\
(\mu \mathrm{T})^{\mathrm{a}}\end{array}$ & Reference \\
\hline \multicolumn{3}{|l|}{ Acceleration of reaction rates } \\
\hline $\mathrm{Na}, \mathrm{K}$-ATPase & $0.2-0.3$ & Blank and Soo [49] \\
\hline cytochrome oxidase & $0.5-0.6$ & Blank and Soo [43] \\
\hline ornithine decarboxylase & $\sim 2$ & Mullins et al. [58] \\
\hline malonic acid oxidation & $<0.5$ & Blank and Soo [59] \\
\hline \multicolumn{3}{|l|}{ Biosynthesis of stress proteins } \\
\hline HL60, Sciara, yeast, & $<0.8$ & Goodman et al. [11] \\
\hline breast (HTB124, MCF7) & $<0.8$ & Lin et al. [39] \\
\hline chick embryo (anoxia) & $\sim 2$ & DiCarlo et al. [60] \\
\hline \multicolumn{3}{|c|}{ Breast cancer (MCF7) cell growth } \\
\hline block melatonin inhibition & $0.2<1.2$ & Liburdy et al. [38] \\
\hline \multirow[t]{2}{*}{ Leukemia epidemiology } & $0.3-4$ & Ahlbom et al. [61] \\
\hline & & Greenland et al. [62] \\
\hline
\end{tabular}

a The estimated values are for departures from the baseline, although Mullins et al. (1999) and DiCarlo et al. (2000) generally give inflection points in the dose-response curves. The leukemia epidemiology values are not experimental and are listed for comparison.

to desensitize the cells to excessive heating during activity. Unlike slow muscle fibers that do synthesize hsp70, cells containing fast muscle fibers do not synthesize hsp70 to protect them from over-reacting to the high temperatures reached a during activity.

\section{EMF-DNA interaction mechanisms: electron transfer}

The biochemical compounds in living cells are composed of charges and dipoles that can interact with electric and magnetic fields by various mechanisms. An example discussed earlier is the generation of reactive oxygen species (ROS) in activation of the ERK signaling cascade. The cellular stress response leading to the synthesis of stress proteins is also activated by EMF. However, the specific reaction is not known, except that it is stimulated by very weak EMF. For this reason, our focus has been on molecular processes that are most sensitive to EMF and that could cause the DNA to come apart to initiate biosynthesis. We have suggested that direct EMF interaction with electrons in DNA is likely for the following reasons:

- The largest effects of EMF would be expected on electrons because of their high charge to mass ratio. At the sub-atomic level, one assumes that electrons respond instantaneously compared to protons and heavier atomic nuclei, as in the Born-Oppenheimer Approximation. The very low field strengths and durations that activate the stress response and other reactions (Table 1) suggest interaction with electrons, and make ion-based mechanisms unlikely.

- Weak ELF fields have been shown to affect the rates of electron transfer reactions [43,44]. A $10 \mu \mathrm{T}$ magnetic field exerts a very small force of only $\sim 10^{-20} \mathrm{~N}$ on a unit charge, 
but this force can move an isolated electron more than a bond length, $\sim 1 \mathrm{~nm}$, in $\sim 1$ nanosecond.

- There is a specific EMF responsive DNA sequence that is associated with the response to EMF (Fig. 1), and that retains this property when transfected

- Displacement of electrons in DNA would cause local charging that has been shown to lead to disaggregation of biopolymers [45].

- As the energy in an EMF stimulus increases, there is an increase in single strand breaks, followed by double strand breaks, suggesting an interaction with EMF at all energy levels [46].

Effects of EMF on electrons in chemical reactions were detected indirectly in studies on the Na,K-ATPase [47], a ubiquitous enzyme that establishes the normal $\mathrm{Na}$ and $\mathrm{K}$ ion gradients across cell membranes. Electric and magnetic fields, each accelerated the reaction only when the enzyme was relatively inactive. It is reasonable to assume that the threshold response occurs when the same charge is affected by the two fields, so the velocity $(v)$ of the charge $(q)$ could be calculated from these measurements and its nature determined. Assuming both fields exert the same force at the threshold, the electric $(E)$ and the magnetic $(B)$ forces should be equal.

$F=q E=q v B$.

From this $v=E / B$, the ratio of the threshold fields, and by substituting the measured thresholds [48,49], $E=5 \times 10^{-4} \mathrm{~V} / \mathrm{m}$ and $B=5 \times 10^{-7} \mathrm{~T}(0.5 \mu \mathrm{T})$, we obtain $v=10^{3} \mathrm{~m} / \mathrm{s}$. This very rapid velocity, similar to that of electrons in DNA [50], indicated that electrons were probably involved in the ion transport mechanism of the Na,K-ATPase [47]. An electron moving at a velocity of $10^{3} \mathrm{~m} / \mathrm{s}$ crosses the enzyme $\left(\sim 10^{-8} \mathrm{~m}\right)$ before the ELF field has had a chance to change. This means that a low frequency sine wave signal is effectively a repeated DC pulse. This is true of all low frequency effects on fast moving electrons.

Studies of effects of EMF on electron transfer in cytochrome oxidase, ATP hydrolysis by the Na,K-ATPase, and the Belousov-Zhabotinski (BZ) redox reaction, have led to certain generalizations:

- EMF can accelerate reaction rates, including electron transfer rates

- EMF acts as a force that competes with the chemical forces in a reaction. The effect of EMF varies inversely with the intrinsic reaction rate, so EMF effects are only seen when intrinsic rates are low. (This is in keeping with the therapeutic efficacy of EMF on injured tissue, while there is usually little or no effect on normal tissue.)

- Experimentally determined thresholds are low $(\sim 0.5 \mu \mathrm{T})$ and comparable to levels found by epidemiology. See Table 1.

- Effects vary with frequency, with different optima for the reactions studied: The two enzymes showed broad fre- quency optima close to the reaction turnover numbers for $\mathrm{Na}, \mathrm{K}$-ATPase $(60 \mathrm{~Hz})$ and cytochrome oxidase $(800 \mathrm{~Hz})$, suggesting that EMF interacted optimally when in synchrony with the molecular kinetics. This is not true for EMF interactions with DNA, which are stimulated in both ELF and RF ranges and do not appear to involve electron transfer reactions with well-defined kinetics.

Probably the most convincing evidence for a frequency sensitive mechanism that involves stimulation of DNA is activation of protein synthesis in striated muscle. In this natural process, specific muscle proteins are synthesized by varying the rate of the (electrical) action potentials in the attached nerves [51]. The ionic currents of the action potentials that flow along and through the muscle membranes, also pass through the muscle cell nuclei that contain the DNA codes for the muscle proteins. Two frequencies were studied in muscle, high $(100 \mathrm{~Hz})$ and low $(10 \mathrm{~Hz})$ frequency, corresponding to the frequencies of the fast muscles and slow muscles that have different contraction rates and different muscle proteins. In the experiments, either the fast or slow muscle proteins were synthesized at the high or low frequency stimulation rates corresponding to the frequency of the action potentials. The clear dependence of the protein composition on the frequency of the action potentials indicates a relation between stimulation and activation of DNA in muscle physiology. The process is undoubtedly far more complicated and unlikely to be a simple electron transfer reaction as with cytochrome oxidase. It is more probable that an entire region of DNA coding for a group of related proteins is activated simultaneously.

A mechanism based on electron movement is in keeping with the $\mathrm{mV} / \mathrm{m}$ electric field and $\mu \mathrm{T}$ magnetic field thresholds that affect the $\mathrm{Na}, \mathrm{K}$-ATPase. The very small force on a charge $\left(\sim 10^{-20} \mathrm{~N}\right)$ can affect an electron, but is unlikely to have a direct effect on much more massive ions and molecules, especially if they are hydrated. Ions are affected by the much larger DC electric fields of physiological membrane processes. The low EMF energy can move electrons, cause small changes in charge distribution and release the large hydration energy tied up in protein and DNA structures [3]. Electrons have been shown to move in DNA at great speed [50], and we have suggested that RF and ELF fields initiate the stress response by directly interacting and accelerating electrons moving within DNA [52,53].

A mechanism based on electron movement also provides insight into why the same stress response is stimulated by both ELF and RF even though the energies of the two stimuli differ by orders of magnitude. A typical ELF cycle at $10^{2} \mathrm{~Hz}$ lasts $10^{-2} \mathrm{~s}$ and a typical RF cycle at $10^{11} \mathrm{~Hz}$ lasts $10^{-11} \mathrm{~s}$. Because the energy is spread over a different number of cycles/second in the two ranges, the energy/cycle is the same in both ELF and RF ranges. Since electron movement occurs much faster than the change of field, both frequencies are seen by rapidly moving electrons as essentially DC pulses. Each cycle contributes to electron movement at both 
frequencies, but more rapidly at the higher frequency. The fluctuation of protons between water molecules in solution at a frequency of about $10^{12} \mathrm{~Hz}$ [54] gives an indication of the speed of electron movement, and may suggest an upper limit of the frequency in which sine wave EMF act as DC pulses.

\section{DNA biology and the EM spectrum}

Research on DNA and the stress response has shown that the same biology occurs across divisions of the EM spectrum, and that EMF safety standards based on cellular measures of potential harm should be much stricter. These data also raise questions about the utility of spectrum sub-divisions as the basis for properly assessing biological effects and setting separate safety standards for the different sub-divisions. The frequencies of the EM spectrum form a continuum, and division into frequency bands is only a convenience that makes it easier to assign and regulate different portions of the spectrum for practical uses, such as the different design requirements of devices for EMF generation and measurement. Except for the special case of the visual range, the frequency bands are not based on biology, and the separate bands now appear to be a poor way of dealing with biological responses needed for evaluating safety. The DNA studies indicate the need for an EMF safety standard rooted in biology and a rational basis for assessing health implications.

DNA responses to EMF can be used to create a single scale for evaluation of EMF dose because:

- The same biological responses are stimulated in ELF and RF ranges.

- The intensity of EMF interactions with DNA leads to greater effects on DNA as the energy increases with frequency. In the ELF range, the DNA is only activated to initiate protein synthesis, while single and double strand breaks occur in the more energetic RF and ionizing ranges.

A scale based on DNA biology also makes possible an approach to a quantitative relation between EMF dose and disease. This can be done by utilizing the data banks that have been kept for A-bomb exposure and victims of nuclear accidents, data that link exposure to ionizing radiation and subsequent development of cancer. Utilizing experimental studies of DNA breaks with ionizing radiation, it is possible in principle to relate cancer incidence to EMF exposures. It should be possible to determine single and double strand breaks in a standard preparation of DNA, caused by exposure to EMF for a specified duration, under standard conditions. Although many studies of DNA damage and repair rates under different conditions would be needed, this appears to be a possible experimental approach to assessing the relation between EMF exposure and disease.

\section{The stress response and safety standards}

Most scientists believe that basic research eventually pays off in practical ways. This has certainly been true of EMF research on the stress response, where EMF stimulated stress proteins have been used to minimize damage to ischemic tissues on reperfusion. However, more importantly, biological effects stimulated by both ELF and RF have shown that the standards used for developing safety guidelines are not protective of cells.

First and foremost, it is important to realize that the stress response occurs in reaction to a potentially harmful environmental influence. The stress response is an unambiguous indication that cells react to EMF as potentially harmful. It is therefore an indication of compromised cell safety, given by the cell, in the language of the cell. The low threshold level of the stress response shows that the current safety standards are much too high to be considered safe.

In general, cellular processes are unusually sensitive to fields in the environment. The biological thresholds in the ELF range (Table 1) are in the range of $0.5-1.0 \mu \mathrm{T}-$ not very much higher than the ELF backgrounds of $\sim 0.1 \mu \mathrm{T}$. The relatively low field strengths that can affect biochemical reactions is a further indication that cells are able to sense potential danger long before there is an increase in temperature.

EMF research has also shown that exposure durations do not have to be prolonged to have an effect. Litovitz et al. [55,56], working with the enzyme ornithine decarboxylase, showed an EMF response when cells were exposed for only $10 \mathrm{~s}$ to ELF or ELF modulated $915 \mathrm{MHz}$, providing that the exposure was continuous. Gaps in the sine wave resulted in a reduced response, and interference with the sine wave in the form of superimposed ELF noise also reduced the response [57]. The interfering effect of noise has been shown in the RF range by Lai and Singh [46], who reported that noise interferes with the ability of an RF signal to cause breaks in DNA strands. The decreased effect when noise is added to a signal is yet another indication that EMF energy is not the critical factor in causing a response. In fact, EMF noise appears to offer a technology for mitigating potentially harmful effects of EMF in the environment.

EMF research has shown that the thermal standard used by agencies to measure safety is at best incomplete, and in reality not protective of potentially harmful non-thermal fields. Non-thermal ELF mechanisms are as effective as thermal RF mechanisms in stimulating the stress response and other protective mechanisms. The current safety standard based on thermal response is fundamentally flawed, and not protective.

Finally, since both ELF and RF activate the same biology, simultaneous exposure to both is probably additive and total EMF exposure is important. Safety standards must consider total EMF exposure and not separate standards for ELF and $\mathrm{RF}$ ranges. 


\section{References}

[1] R. Goodman, C.A.L. Bassett, A. Henderson, Pulsing electromagnetic fields induce cellular transcription, Science 220 (1983) 12831285.

[2] R. Goodman, A. Henderson, Exposure of salivary gland cells to low frequency electromagnetic fields alters polypeptide synthesis, PNAS 85 (1988) 3928-3932.

[3] M. Blank, Protein and DNA interactions with electromagnetic fields, Electromagn Biol Med 28 (2008) 3-23.

[4] W.R. Adey, Tissue interactions with non-ionizing electromagnetic fields, Physiol Rev 61 (1981) 435-514.

[5] H. Lin, M. Blank, R. Goodman, A magnetic field responsive domain in the human HSP70 promoter, J Cell Biochem 75 (1999) 170-176.

[6] H. Lin, M. Blank, K. Rossol-Haseroth, R. Goodman, Regulating genes with electromagnetic response elements, J Cell Biochem 81 (2001) 143-148.

[7] REFLEX Project Report, 2004, A summary of the final report can be found at http://www.verum-foundation.de/www2004/html/pdf/ euprojekte01/REFLEX_ProgressSummary_231104.pdf.

[8] H. Lai, N.P. Singh, Acute exposure to a $60 \mathrm{~Hz}$ magnetic field increases DNA strand breaks in rat brain cells, Bioelectromagnetics 18 (1997) 156-165.

[9] Bioinitiative Report, edited by C. Sage, D. Carpenter, A Scientific Perspective on Health Risk of Electromagnetic Fields, published online 31 August $2007 \mathrm{http} / / / \mathrm{www}$.bioinitiative.org/report/index.htm.

[10] M. Blank, O. Khorkova, R. Goodman, Changes in polypeptide distribution stimulated by different levels of EM and thermal stress, Bioelectrochem Bioenerg 33 (1994) 109-114.

[11] R. Goodman, M. Blank, H. Lin, O. Khorkova, L. Soo, D. Weisbrot, A.S. Henderson, Increased levels of hsp70 transcripts are induced when cells are exposed to low frequency electromagnetic fields, Bioelectrochem Bioenerg 33 (1994) 115-120.

[12] D. Kultz, Molecular, Evolutionary basis of the cellular stress response, Ann Rev Physiol 67 (2005) 225-257.

[13] R. Goodman, M. Blank, Magnetic field stress induces expression of hsp70, Cell Stress Chaperones 3 (1998) 79-88.

[14] F.M. Ritossa, A new puffing pattern induced by a temperature shock and DNP in Drosophila, Experientia Basel 18 (1962) 571-573.

[15] D.I. de Pomerai, C. Daniells, H. David, J. Allan, I. Duce, M. Mutwakil, D. Thomas, P. Sewell, J. Tattersall, D. Jones, Non-thermal heat-shock response to microwaves, Nature 6785 (2000) 417-418.

[16] J. Czyz, K. Guan, Q. Zeng, T. Nikolova, A. Meister, F. Schönborn, I. Schuderer, N. Kuster, A.M. Wobus, High frequency electromagnetic fields (GSM signals) affect gene expression levels in tumor suppressor p53-deficient embryonic stem cells, Bioelectromagnetics 25 (2004) 296-307.

[17] T. Taira, Y. Negishi, F. Kihara, S.M.M. Iguchi-Ariga, H. Ariga, H. c-myc protein complex binds to two sites in human hsp70 promoter region, Biochim Biophys Acta 1130 (1992) 166-174.

[18] J. Topol, D.M. Ruden, C.S. Parker, Sequences required for in vitro transcriptional activation of a drosophila hsp70 gene, Cell 42 (1985) 527-537.

[19] A. Albertini, B. Zucchini, G. Noera, R. Cadossi, C.P. Napoleone, A. Pierangeli, Protective effect of low frequency low energy policy electromagnetic fields on acute experimental myocardial infarcts in rats, Bioelectromagnetics 20 (1999) 372-377.

[20] A. Di Carlo, J.M. Farrell, T. Litovitz, A simple experiment to study electromagnetic field effects: protection induced by short-term exposures to $60 \mathrm{~Hz}$ magnetic fields, Bioelectromagnetics 19 (1998) 498500.

[21] J.M. Shallom, A.L. DiCarlo, D. Ko, L.M. Penafiel, A. Nakai, Microwave exposure induces hsp70 and confers protection against hypoxia in chick embryos, J Cell Biochem 86 (2002) 490-496.

[22] I. George, M. Geddis, Z. Lill, H. Lin, T. Gomez, M. Blank, M. Oz, R. Goodman, Myocardial function improved by electromagnetic fields induction of stress protein hsp70, J Cellular Physiol 216 (2008) 816-823, published online: doi:10.1002/jcp.21461.

[23] J. Friedman, S. Kraus, Y. Hauptman, Y. Schiff, R. Seger, Mechanism of short-term ERK activation by electromagnetic fields at mobile phone frequencies, Biochem J 405 (2007) 559-568.

[24] M. Jin, M. Blank, R. Goodman, ERK1/2 phosphorylation, induced by electromagnetic fields, diminishes during neoplastic transformation, $\mathrm{J}$ Cell Biochem 78 (2000) 371-379.

[25] D. Leszczynski, S. Joenvaara, J. Reivinen, R. Kuokka, Non-thermal activation of the hsp27/p38MAPK stress pathway by mobile phone radiation in human endothelial cells: molecular mechanism for cancer-and blood-brain barrier-related effects, Differentiation 70 (2002) 120-129.

[26] M. Simko, Induction of cell activation processes by low frequency electromagnetic fields, ScientificWorldJournal 4 (Suppl. 2) (2004) 4-22.

[27] R. Marais, J. Wynne, R. Treisman, The SRF accessory protein Elk-1 contains a growth factor-regulated transcriptional activation domain, Cell 73 (1993) 381-393.

[28] R. Janecht, W.H. Ernst, V. Pigoud, A. Nordheim, Activation of TCF Elk-1 by MAP kinases, EMBO J 12 (1993) 5097-5104.

[29] H. Gille, M. Kortenjann, O. Thomae, C. Moomaw, C. Slaughter, M.H. Cobb, P.E. Shaw, ERK phosphorylation potentiates Elk-1-mediated ternary complex formation and transactivation, EMBO J 14 (1995) 951-962.

[30] V. Sgambato, P. Vanhouttte, C. Pages, M. Rogard, L. Hipskind, M.J. Besson, J. Caboche, In vivo expression and regulation of Elk-1, a target of the extracellular-regulated kinase signaling pathway, in the rat brain, J. Neurosci 18 (1998) 214-226.

[31] D. Leszczynski, R. Nylund, S. Joevaara, J. Reivinen, Applicability of discovery science approach to determine biological effects of mobile phone radiation, Proteonomics 4 (2004) 426-431.

[32] G.J. Hook, P. Zhang, I. Lagroye, L.E. Li, R. Higashikubo, E.G. Moros, W.L. Starube, W.F. Pickard, J.D. Baty, J.L. Roti-Roti, Measurement of DNA damage and apoptosis in Molt-4 cells after in vitro exposure to radio frequency radiation, Radiat Res 161 (2004) 193-200.

[33] D. Weisbrot, H. Lin, L. Ye, M. Blank, R. Goodman, Effects of mobile phone radiation on growth and development in Drosophila melanogaster, J Cell Biochem 89 (2003) 48-55.

[34] S. Rao, A.S. Henderson, Regulation of c-fos is affected by electromagnetic fields, J Cell Biochem 63 (1996) 358-365.

[35] D.L. Johnson, S.A.S. Johnson, RNA metabolism and oncogenesis, Science 320 (2008) 461-462.

[36] I.A. Cotgreave, Biological stress responses to radio frequency electromagnetic radiation: are mobile phones really so (heat) shocking? Arch Biochem Biophys 435 (2005) 227-240.

[37] M. Jin, H. Lin, L. Han, M. Opler, S. Maurer, M. Blank, R. Goodman, Biological and technical variables in myc expression in HL60 cells exposed to $60 \mathrm{~Hz}$ electromagnetic fields, Bioelectrochem Bioenerg 44 (1997) 111-120.

[38] R.P. Liburdy, T.R. Sloma, R. Sokolic, P. Yaswen, ELF magnetic fieklds, breast cancer, and melatonin: $60 \mathrm{~Hz}$ fields block melatonin's oncostatic action on ER+ breast cancer cell proliferation, J Pineal Res 14 (1993) 89-97.

[39] H. Lin, M. Head, M. Blank, L. Han, M. Jin, R. Goodman, Myc-mediated transactivation of HSP70 expression following exposure to magnetic fields, J Cell Biochem 69 (1998) 181-188.

[40] S. Ivancsits, A. Pilger, F. Diem, O. Jahn, H. Rudiger, Cell type-specific genotoxic effects of intermittent extremely low-frequency electromagnetic fields, Mutation Res 583 (2005) 184-188.

[41] M. Lantow, M. Lupke, J. Frahm, M.O. Mattsson, N. Kuster, M. Simko, ROS release and Hsp70 expression after exposure to $1,800 \mathrm{MHz}$ radiofrequency electromagnetic fields in primary human monocytes and lymphocytes, Radiat Environ Biophys 45 (2006) 55-62.

[42] M. Simko, C. Hartwig, M. Lantow, M. Lupke, M.O. Mattsson, Q. Rahman, J. Rollwitz, Hsp70 expression and free radical release after exposure to non-thermal radio-frequency electromagnetic fields and ultrafine particles in human Mono Mac 6 cells, Toxicol Lett 161 (2006) 73-82. 
[43] M. Blank, L. Soo, Enhancement of cytochrome oxidase activity in 60 $\mathrm{Hz}$ magnetic fields, Bioelectrochem Bioenerg 45 (1998) 253-259.

[44] M. Blank, L. Soo, Electromagnetic acceleration of the BelousovZhabotinski reaction, Bioelectrochem 61 (2003) 93-97.

[45] M. Blank, L. Soo, Surface free energy as the potential in oligomeric equilibria: prediction of hemoglobin disaggregation constant, Bioelectrochem Bioenerg 17 (1987) 349-360.

[46] H. Lai, N.P. Singh, Interaction of microwaves and a temporally incoherent magnetic field on single and double DNA strand breaks in rat brain cells, Electromagn Biol Med 24 (2005) 23-29.

[47] M. Blank, A proposed explanation for effects of electric and magnetic fields on the Na,K-ATPase in terms of interactions with electrons, Bioelectromagnetics 26 (2005) 591-597.

[48] M. Blank, L. Soo, The threshold for alternating current inhibition of the Na,K-ATPase, Bioelectromagnetics 13 (1992) 329-333.

[49] M. Blank, L. Soo, The threshold for Na,K-ATPase stimulation by electromagnetic fields, Bioelectrochem Bioenerg 40 (1996) 63-65.

[50] C. Wan, T. Fiebig, S.O. Kelley, C.R. Treadway, J.K. Barton, Femtosecond dynamics of DNA-mediated electron transfer, Proc Nat Acad Sci U S A 96 (1999) 6014-6019.

[51] M. Blank, Electric stimulation of protein synthesis in muscle, Adv Chem 250 (1995) 143-153.

[52] M. Blank, R. Goodman, Initial interactions in electromagnetic fieldinduced biosynthesis, J Cell Physiol 199 (2004) 359-363.

[53] M. Blank, R. Goodman, A mechanism for stimulation of biosynthesis by electromagnetic fields: charge transfer in DNA and base pair separation, J Cell Physiol 214 (2008) 20-26.
[54] C.J. Fecko, J.D. Eaves, J.J. Loparo, A. Tokmakoff, P.L. Geissler, Ultrafast hydrogen-bond dynamics in infrared spectroscopy of water, Science 301 (2003) 1698-1701.

[55] T.A. Litovitz, D. Kraus, J.M. Mullins, Effect of coherence time of the applied magnetic field on ornithine decarboxylase activity, Biochem Biophys Res Comm 178 (1991) 862-865.

[56] T.A. Litovitz, D. Kraus, M. Penafiel, E.C. Elson, J.M. Mullins, The role of coherence time in the effect of microwaves on ornithine decarboxylase activity, Bioelectromagnetics 14 (1993) 395-403.

[57] J.M. Mullins, T.A. Litovitz, M. Penafiel, A. Desta, A. Krause, Intermittent noise affects EMF- induced ODC activity, Bioelectrochem Bioenerg 44 (1998) 237-242.

[58] J.M. Mullins, L.M. Penafiel, J. Juutilainen, T.A. Litovitz, Doseresponse of electromagnetic field-induced ornithine decarboxylase activity, Bioelectrochem Bioenerg 48 (1999) 193-199.

[59] M. Blank, L. Soo, Electromagnetic acceleration of electron transfer reactions, J Cell Biochem 81 (2001) 278-283.

[60] A.L. Di Carlo, J.M. Mullins, T.A. Litovitz, Thresholds for EM fieldinduced hypoxia protection: evidence for a primary, electric field effect, Bioelectrochem 52 (2000) 9-16.

[61] H. Ahlbom, N. Day, M. Feychting, E. Roman, J. Skinner, J. Dockerty, M. Linet, M. McBride, J. Michaelis, J.H. Olsen, T. Tynes, P.K. Verkasalo, A pooled analysis of magneticfields and childhood leukemia, Brit J Cancer 83 (2000) 692-698.

[62] S. Greenland, A.R. Sheppard, W.T. Kaune, C. Poole, M.A.A. Kelsh, Pooled Analysis of Magnetic Fields, Wire Codes, and Childhood Leukemia, Epidemiology 11 (2000) 624-634. 